\title{
ON HOMOGENEOUS SPACES, HOLONOMY, AND NON-ASSOCIATIVE ALGEBRAS
}

\author{
ARTHUR A. SAGLE*
}

1. Introduction. Let $G$ be a connected Lie group and $H$ a closed subgroup. The homogeneous space $M=G / H$ is called reductive if in the Lie algebra $g$ of $G$ there exists a subspace $\mathfrak{m}$ such that $\mathfrak{g}=\mathfrak{m}+\mathfrak{h}$ (subspace direct sum) and $[\mathfrak{h m}] \subset \mathfrak{m}$ where $\mathfrak{h}$ is the Lie algebra of $H$, see [8]. In this case the pair $(\mathfrak{g}, \mathfrak{h})$ is called a reductive pair and the subspace $\mathfrak{m}$ can be made into an anti-commutative algebra as follows. For $X, Y \in \mathfrak{m}$ let $[X Y]$ $=X Y+h(X, Y)$ where $X Y=[X Y]_{\mathfrak{m}}$ (resp. $\left.h(X, Y)=[X Y]_{\mathfrak{h}}\right)$ is the projection of $[X Y]$ in $\mathfrak{g}$ into $\mathfrak{m}$ (resp. $\mathfrak{h})$. The Lie algebra identities of $\mathfrak{g}$ yield the following identities for $\mathfrak{m}$ and $\mathfrak{h}$. For $X, Y, Z \in \mathfrak{m}$ and $h \in \mathfrak{h}$,

(1) $X Y=-Y X \quad$ (bilinear)

(2) $h(X, Y)=-h(Y, X) \quad$ (bilinear)

(3) $[Z h(X, Y)]+[X h(Y, Z)]+[Y h(Z, X)]=(X Y) Z+(Y Z) X+(Z X) Y$

(4) $h(X Y, Z)+h(Y Z, X)+h(Z X, Y)=0$

(5) $[h \quad h(X, Y)]=h\left(\left[\begin{array}{ll}h X & X, Y)+h(X,[h Y])\end{array}\right.\right.$

(6) $\left[\begin{array}{ll}h & X Y\end{array}\right]=\left[\begin{array}{ll}h & X\end{array}\right] Y+X\left[\begin{array}{ll}h & Y\end{array}\right]$.

The above algebra will be denoted by $(\mathfrak{m}, X Y)$ and note that (6) says the mappings $D(h)=a d_{\mathfrak{m}} h: \mathfrak{m} \rightarrow \mathfrak{m}: X \rightarrow[h X]$ are derivations of this algebra. Also note $G / H$ is a symmetric space if $(\mathfrak{m}, X Y)$ is the zero algebra.

The algebra $(\mathfrak{m}, X Y)$ and other non-associative algebras are related to the differential geometry of $G / H$ by using the following results of Nomizu [8, Th. 8. 1].

THEOREM. Let $G / H$ be a reductive homogeneous space with a fixed Lie algebra decomposition $\mathfrak{g}=\mathfrak{m}+\mathfrak{h}$ with $[\mathfrak{h} \mathfrak{m}] \subset \mathfrak{m}$. Then there exists a one-to-one correspondence between the set of all $G$-invariant connections on $G / H$ and the set of all bilinear functions $\alpha: \mathfrak{m} \times \mathfrak{m} \rightarrow \mathfrak{m}$ such that the mappings $D(h)$ are derivations of the resulting algebra.

Received July 17, 1967.

* Supported in part by N.S.F. Grant GP-6364 and GP-7209. 


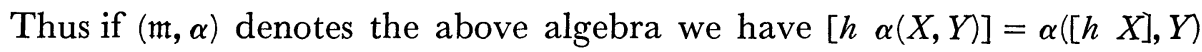
$+\alpha\left(X,\left[\begin{array}{ll}h & Y\end{array}\right]\right)$. From [8, section 9] we have the following formulas for the curvature endomorphism and torsion in terms of $(\mathfrak{m}, \alpha)$. Motivated by the notation in [5], let $a(X): \mathfrak{m} \rightarrow \mathfrak{m}: Y \rightarrow \alpha(X, Y)$ and $b(X): \mathfrak{m} \rightarrow \mathfrak{m}: Y \rightarrow \alpha(Y, X)$, then

(7) $R(X, Y)=[a(X), a(Y)]-a(X Y)-D(h(X, Y))$,

(8) Tor $(X, Y)=[a(X)-b(X)-L(X)] Y$

where $L(X): \mathfrak{m} \rightarrow \mathfrak{m}: Y \rightarrow X Y$.

From [8, Th. 10.1] the particular algebra $(\mathfrak{m}, X Y)$ is further related to the geometry of $G / H$ as follows.

TheOREm. On a reductive homogeneous space $G / H$, there exists one and only one $G$-invariant connection which has zero torsion and such that a 1-parameter subgroup $x(t)$ in $G$ generated by $X \in \mathfrak{m}$ projects by $\pi: G \rightarrow G / H: x(t) \rightarrow x^{*}(t)$ into a geodesic $x^{*}(t)$ in $G / H$. In this case $\alpha(X, Y)=\frac{1}{2} X Y$ and the connection is called the (canonical) connection of the first kind on $\mathrm{G} / \mathrm{H}$.

Using identities (1)-(6) various results were proved in $[10 ; 11 ; 12 ; 13]$ relating the algebra $(\mathfrak{m}, X Y)$ to the geometry of $G / H$. In this paper we attempt to extend some of these results to the general connection on $G / H$ defined by $(\mathfrak{n t}, \alpha)$ by comparing it with the algebra $(\mathfrak{m}, X Y)$. A comparison theory was developed by Kostant in [5].

Given a connection on $M=G / H$ defined by the algebra (m, $\alpha$ ), the corresponding holonomy group, $\mathrm{Hol}(\alpha)$, acts on $M$ by $G$-translating its action on the tangent space $M_{p}=\mathfrak{m}$ to all of $M(p=H \in M)$. Thus since $\mathrm{Hol}(\alpha)$ is a Lie group, we shall consider its Lie algebra, hol $(\alpha)$, called the holonomy algebra as a Lie algebra of endomorphisms acting on $\mathfrak{m}$. From $[5 ; 7 ; 9]$ we see that $\operatorname{hol}(\alpha)$ is the smallest Lie algebra $\mathfrak{h}^{*}$ such that all $R(X, Y) \in \mathfrak{h}^{*}$ and $\left[a(X), \mathfrak{h}^{*}\right] \subset \mathfrak{h}^{*}$. Thus $\mathfrak{h}^{*}=\operatorname{hol}(\alpha)$ is generated by $\{a(X)$ and $D(h(X, Y)): X, Y \in \mathfrak{m}\}$. Geometrically, holonomy irreducible spaces $G / H$ are first considered and then extended by a "de Rham decomposition theorem" to holonomy "completely reducible" spaces [10, Th. 8]. Algebraically, this leads to new problems not only in simple algebras ( $m, \alpha)$ but also in algebras $(\mathfrak{m}, \alpha)$ which have no $\mathfrak{h}^{*}$-invariant subspaces; i.e. no left ideals which are invariant under the derivation algebra.

In section 2 we review some basic facts in terms of the above nonassociative algebras. We see that the algebras $(\mathfrak{m}, \alpha)$ generalize Lie admissible algebras to reductive Lie admissible algebras [11, section 1]. 
Next we see that holonomy irreducibility implies $(\mathfrak{n t}, \alpha)$ is a simple algebra, but frequently this algebra need not have an identity or idempotent elements e.g. $G / H$ Riemannian. Following the lead in [5], we compare a pseudo-Riemannian connection given by $(\mathfrak{m}, \alpha)$ with a pseudo-Riemannian connection of the first kind; we do this comparison by a one-to-one correspondence between algebras $(\mathfrak{m}, \alpha)$ and elements in a neighborhood of the identity in a certain Jordan algebra.

In section 3 we give some applications of algebras $(\mathfrak{m}, \alpha)$. For example, we show that if $(\mathfrak{g}, \mathfrak{h})$ is a reductive pair with decomposition $\mathfrak{g}=\mathfrak{m}+\mathfrak{h}$ such that $D(\mathfrak{h})$ acts irreducible on $\mathfrak{m}$, then any algebra $(\mathfrak{m}, \alpha)$ must induce an irreducible connection of the first kind [16]. We extend this using the algebra $(\mathfrak{m}, \alpha)$ to prove Kostant's results [5] about $D(\mathfrak{h})$ having inequivalent representations in $\mathfrak{m}$ implying $G / H$ is metric irreducible. We also give an example of a reductive pair $(\mathfrak{g}, \mathfrak{h})$ with $\mathfrak{g}$ simple and compact which yields reducible or irreducible connections. This example seems to indicate that the problem of irreducibility in the general case is more numerical than structural; compare with the results on the connection of the first kind in [13].

In section 4 we introduce the Lie transformation algebra $L(\alpha)$ generated by all the endomorphisms $a(X), b(Y)$ for $X, Y \in \mathfrak{m}$. We show that if $G / H$ is a $\mathrm{Hol}(\boldsymbol{\alpha})$-irreducible pseudo-Riemannian manifold, then hol $(\alpha)$ is a reductive subalgebra of $L(\alpha)$ and in this case it is easy to see that $L(\alpha)$ is the Lie algebra of the Lie group generated by $\operatorname{Hol}(\alpha)$ and the holonomy group of the connection of the first kind. We also note $L(\alpha)=\operatorname{hol}(\alpha)$ if and only if $(\mathfrak{m}, \alpha)$ induces a connection of the first kind. Finally when $g$ is semi-simple (with $G / H$ irreducible as above), we show how to reduce the study of a pseudo-Riemannian connection induced by $(\mathfrak{m}, \alpha)$ to the study of a finite sequence of reductive pairs $\left(L_{i}, H_{i}\right)$ where $L_{i}$ is semi-simple and $\left(L_{i}, H_{i}\right)$ is an irreducible symmetric pair or induces an irreducible connection of the first kind on the corresponding homogeneous space $\mathscr{L}_{i} / \mathscr{H}_{i}$.

2. Algebra $(\mathfrak{m}, \boldsymbol{a})$. Let $(\mathfrak{g}, \mathfrak{h})$ be a reductive pair with fixed decomposition $\mathfrak{g}=\mathfrak{m}+\mathfrak{h}$ and $[\mathfrak{h} \mathfrak{m}] \subset \mathfrak{m}$. Then as previously described a $G$ invariant connection on $G / H$ yields a non-associative algebra (m, $\alpha$ ) so that $D(\mathfrak{h})=a d_{\mathfrak{m}} \mathfrak{h}$ are derivations of this algebra. We shall assume that $G / H$ is simply connected then in this case $[7 ; 10]$, the holonomy group, $\operatorname{Hol}(\alpha)$, 
is irreducible in $\mathfrak{m}$ if and only if its Lie algebra, hol $(\alpha)$, is irreducible in $\mathfrak{m}$. We shall also assume $G / H$ is not "flat" i.e. $\operatorname{hol}(\alpha) \neq 0$.

Next we note that if $\mathrm{hol}(\alpha)$ is reducible, then there is a $D(\mathfrak{h})$-invariant subspace $\mathfrak{n}$ of $\mathfrak{m}$ which is $a(\mathfrak{m})$-invariant; i.e. $\mathfrak{n}$ is a $D(\mathfrak{h})$-invariant left ideal of the algebra $(\mathfrak{m}, \alpha)$. From this remark we obtain the following theorem.

THEOREM 1. Let $G / H$ be a simply connected reductive homogeneous space with fixed decomposition $\mathfrak{g}=\mathfrak{m}+\mathfrak{h}$ such that the algebra $(\mathfrak{m}, \alpha)$ determines a $G$ invariant connection. If $G / H$ is a holonomy irreducible non-symmetric space with zero torsion, then the algebra $(\mathfrak{m}, \alpha)$ is simple.

Proof. First $(\mathfrak{m}, \alpha)$ is not the zero algebra; for if $\alpha(X, Y)=0$ for all $X, Y \in \mathfrak{m}$, then $0=\operatorname{Tor}(X, Y)=\alpha(X, Y)-\alpha(Y, X)-X Y=X Y$. Thus $G / H$ would be symmetric. Next if $(\mathfrak{m}, \alpha)$ has a proper ideal $\mathfrak{b}$ (i.e. subspace $\mathfrak{b}$ with $\alpha(\mathfrak{b}, \mathfrak{m}) \subset \mathfrak{b}$ and $\alpha(\mathfrak{m}, \mathfrak{b}) \subset \mathfrak{b})$, then $(\mathfrak{m}, \alpha)$ has a proper ideal $\mathfrak{n}$ which is $D(\mathfrak{h})$-invariant $[10 ; 11 ; 13]$. Thus $\operatorname{hol}(\alpha) \mathfrak{n} \subset \mathfrak{n}$ so that $G / H$ is not holonomy irreducible, a contradiction.

Contrary to the situation of a connection of the first kind, the converse of this theorem is false. In [5, section 3. 2] Kostant constructs a simply connected reductive non-symmetric homogeneous space $G / H$ with $G$ simple and compact such that $G / H$ has a reducible ( $G$-invariant) metric connection induced from the Killing form in $\mathfrak{g}$. Thus in the decomposition $\mathfrak{g}=\mathfrak{m}+\mathfrak{h}$ we obtain an algebra $(\mathfrak{m}, \alpha)$ which induces this reducible connection. This algebra is simple; for if $\mathfrak{b}$ is an ideal of this algebra, then since the connection is metric we have for $X \in \mathfrak{m}, Y \in \mathfrak{b}$ that $X Y=\alpha(X, Y)-\alpha(Y, X)$ $\in \mathfrak{b}$. Thus the algebra $(\mathfrak{m}, X Y)$ has a proper ideal so that $G / H$ is not irreducible relative to the connection of the first kind [10]. This contradicts the fact that if $G$ is simple and $G / H$ is reductive Riemannian simply connected homogeneous space, then $G / H$ is irreducible relative to the connection of the first kind [10].

The algebras $(\mathfrak{m}, \alpha)$ are not as general as might be expected. First as explained in [11] the algebra $(\mathfrak{m}, \alpha)$ is reductive Lie admissible when Tor $(X, Y)=0$ as follows. In general a non-associative algebra $A$ with multiplication $X Y$ yields an algebra $A^{-}$which is the same vector space $A$ but with multiplication $X^{*} Y=X Y-Y X$. The algebra $A$ is reductive Lie admissible if there is a Lie subalgebra, $D\left(A^{-}\right)$, of the derivation algebra of $A^{-}$such that $\mathfrak{g}=A^{-} \oplus D\left(A^{-}\right)$is a Lie algebra and $\left(\mathfrak{g}, D\left(A^{-}\right)\right)$is a reductive pair relative to the above decomposition and $[X Y]_{A^{-}}=X^{*} Y$. Note that 
if $D\left(A^{-}\right)$can be taken to be 0 , then $A$ is Lie admissible; in general a non-associative algebra $A$ is Lie admissible if $A^{-}$is a Lie algebra; see referençes in [11]. Also in [11;17] it is shown that alternative algebras are reductive Lie admissible and therefore so are associative algebras. It should be noted that Jordan algebras arise from symmetric pairs $(\mathfrak{g}, \mathfrak{h})$ and are trivially Lie admissible.

Next we note that the reductive Lie admissible algebra $(\mathfrak{m}, \alpha)$ is a subspace of a Lie admissible algebra as follows. Let $A$ be the vector space $\mathfrak{m} \dot{+} \mathfrak{h}=\mathfrak{g}$ and define multiplication on $A$ by:

(a) $X, Y \in \mathfrak{m}, \quad$ then $X \cdot Y=\alpha(X, Y)+\frac{1}{2} h(X, Y)$;

(b) $X \in \mathfrak{m}$ and $h \in \mathfrak{h}$, then $2 X \cdot h=\left[\begin{array}{ll}X & h\end{array}\right]$ in the Lie algebra $\mathfrak{g}$;

(c) $h_{1} \in \mathfrak{h}$ and $h_{2} \in \mathfrak{h}$, then $2 h_{1} \cdot h_{2}=\left[\begin{array}{ll}h_{1} & h_{2}\end{array}\right]$ in the Lie algebra $g$.

Then with this multiplication $A$ is a Lie admissible algebra such that $A^{-}=\mathrm{g}$ as Lie algebras. Note that for $\alpha(X, Y)=\frac{1}{2} X Y$ i.e. canonical connection of first kind with zero torsion, we have $A=\mathrm{g}$. The role of the algebra $A$ in studying the connection determined by $(\mathfrak{m}, \alpha)$ is probably analogous to the Lie algebra $g$ and the connection of the first kind. For example, analogous to the results concerning a simple Lie algebra $g$ implying $G / H$ is irreducible relative to the connection of the first kind [10], one can ask if $A$ is simple, then when is $G / H$ irreducible relative to the connection induced by $(\mathfrak{m}, \alpha)$.

We now study the algebra $(\mathfrak{m}, \alpha)$ in more detail when it induces a pseudo-Riemannian connection on $G / H$. Thus from $[8 ; 5]$ we see that the reductive pair $(\mathfrak{g}, \mathfrak{h})$ with fixed decomposition $\mathfrak{g}=\mathfrak{m}+\mathfrak{h}$ is such that there is a nondegenerate symmetric bilinear form, $C$, defined on $\mathfrak{m}$ which satisfies

$$
C(D X, Y)=-C(X, D Y)
$$

for $D=a d_{\mathfrak{m}} h$ with $h \in \mathfrak{h}$ and

$$
C(\alpha(Z, X), Y)=-C(X, \alpha(Z, Y))
$$

for $X, Y, Z \in \mathfrak{m}$. Thus the mappings $D$ and $a(Z)$ are $C$-skew-symmetric. Also from [8, section 13] the multiplication $\alpha(X, Y)$ can be written uniquely as 


$$
\alpha(X, Y)=\frac{1}{2} X Y+U(X, Y)
$$

where $U(X, Y)$ is a commutative multiplication on $\mathfrak{m}$ and might be regarded as a perturbation of the connection of the first kind (given by $\frac{1}{2} X Y$ ) to obtain the connection given by $\alpha(X, Y)$. Note that $\alpha(X, Y)-\alpha(Y, X)=X Y$; this is because Tor $(X, Y)=0$.

Also from [8] we have that $U(X, Y)$ is uniquely determined by the form $C$ and this yields the unique determination of $\alpha(X, Y)$ by

$$
2 C(Z, \alpha(X, Y))=C(Z, X Y)+C(Z X, Y)+C(X, Z Y) .
$$

We have seen that when $(\mathfrak{m}, \alpha)$ induces an irreducible connection, this algebra is simple. However, contrary to the usual simple algebras, these algebras need not have an identity or idempotent elements. Let ( $\mathfrak{m}, \alpha)$ induce an irreducible pseudo-Riemannian connection via the nondegenerate form $C$ and suppose $e$ is the identity element for $(\mathfrak{m}, \alpha)$. Then $X=\alpha(e, X)$ $=\alpha(X, e)+e X=X+e X$; thus $e X=0$ for any $X \in \mathfrak{m}$. Next using (9) we have

$$
\begin{aligned}
2 C(e, X) & =2 C(e, \alpha(e, X)) \\
& =C(e, e X)+C(e e, X)+C(e, e X) \\
& =0 ;
\end{aligned}
$$

thus $e=0$, a contradiction. Next if the connection is Riemannian and if $e=\alpha(e, e)$ is an idempotent of $(\mathfrak{m}, \alpha)$, then using (9),

$$
2 C(e, e)=2 C(e, \alpha(e, e))=0 ;
$$

thus in this case $(\mathfrak{m}, \alpha)$ cannot have an idempotent.

Proposition 2. Let $(\mathfrak{g}, \mathfrak{h})$ be a reductive pair with fixed decomposition $\mathrm{g}=\mathfrak{m} \dot{+} \mathfrak{h}$ which induces an irreducible pseudo-Riemannian connection on the corresponding $G / H$ by means of the algebra $(\mathfrak{m}, \alpha)$. Then $(\mathfrak{m}, \alpha)$ is not an alternative algebra (and therefore not associative).

Proof. We use the fact that a simple finite dimensional alternative algebra over the reals has an identity. Thus although alternative algebras yield connections, they are not irreducible.

In [5] Kostant compares the general metric connection with the connection of the first kind when it is also a metric connection. Many of these 
results extend to pseudo-Riemannian connections and we shall do a similar comparison of the connections and the holonomy algebras by means of the algebras defined on $\mathrm{m}$.

For a fixed decomposition $\mathfrak{g}=\mathfrak{m}+\mathfrak{h}$ let the algebra $\mathfrak{m}$ with multiplication $X Y=\left[\begin{array}{lll}X & Y\end{array}\right]_{\mathfrak{m}}$ be denoted by $(\mathfrak{m}, X Y)$. Let the canonical connection of the first kind be induced by the pseudo-Riemannian metric $B(X, Y)$; thus the algebra $(\mathfrak{m}, X Y)$ is an anti-commutative algebra with an invariant form [10] and the mappings $D(h)$ and $L(Z): m \rightarrow m: X \rightarrow Z X$ are B-skew-symmetric. As in [5] we have, since $B$ and $C$ are nondegenerate forms on $\mathfrak{m}$, that there is $S \in G L(\mathfrak{m})$ such that $C(X, Y)=B(S X, Y)$ for $X, Y \in \mathfrak{m}$. Thus from (9) we see the algebras $(\mathfrak{m}, \alpha)$ and $(\mathfrak{m}, X Y)$ are related by

$$
2 \alpha(X, Y)=X Y+S^{-1}[X(S Y)-(S X) Y]
$$

that is, $U(X, Y)=\frac{1}{2} S^{-1}[X(S Y)-(S X) Y]$.

Some immediate properties of $S$ are

(a) $[S, D]=0 \quad$ for all $D \in a d_{\mathfrak{m}} \mathfrak{h}$ and

(b) $S^{b}=S^{c}=S$

where $b$ and $c$ denote adjoints relative to $B$ and $C$. Conversely if $S^{b}$ $=S \in G L(\mathfrak{m})$ satisfies (a), then we can define a pseudo-Riemannian metric $C$ and a corresponding connection by the above formulas. Next note that the set of $S^{\prime} s$ in Hom $(\mathfrak{m}, \mathfrak{m})$ satisfying (a) and (b) form a Jordan algebra $(A, \cdot)$ under the usual multiplication $2 S_{1} \cdot S_{2}=S_{1} S_{2}+S_{2} S_{1}$. The $S$ 's which yield connections are the elements of $M=A \cap G L(\mathfrak{m})$, which is a submanifold of $A$ and forms a reflexion space [6]. Thus for $L(S): A \rightarrow A: X \rightarrow S \cdot X$ and for $Q(S)=2 L(S)^{2}-L\left(S^{2}\right)$ we have that $M$ is closed under the differentiable product

$$
S_{1} * S_{2}=Q\left(S_{1}\right) S_{2}^{-1} \text {. }
$$

More details will appear in a later paper.

3. Some applications. For the applications of the algebras $(\mathfrak{m}, \alpha)$ and $(\mathfrak{m}, X Y)$ we shall assume that the reductive pair $(\mathfrak{g}, \mathfrak{h})$ and the (fixed) decomposition $g=\mathfrak{m}+\mathfrak{h}$ is such that $D(h)=0$ implies $h=0$. First let $D(h)=a d_{\mathfrak{m}} h$ act irreducibly in $\mathfrak{m}$, then from [10, Th. 6] we have $\mathfrak{m m}=0$ or $(\mathfrak{m}, X Y)$ is simple; thus $G / H$ is an irreducible symmetric space or $G / H$ is 
irreducible non-symmetric space relative to the connection of the first kind. Now assume the connection of the first kind is given by a pseudo-Riemannian metric $B$. For example, if $\mathfrak{g}$ is simple, then using [11, Th. 6] $\mathfrak{h}$ is semi-simple so that we may take $\mathfrak{m}=\mathfrak{h}^{\perp}$ relative to the Killing form of $\mathfrak{g}$ and let $B$ equal the Killing form restricted to $\mathfrak{m} \times \mathfrak{m}$, see [13]. Now let $(\mathfrak{m}, \alpha)$ be any other algebra where $\alpha$ is given by (10) and induced by a metric $C$. Since $S=S^{c}$ and $C$ is a metric, $S$ has a real characteristic value $\lambda$. Since $[S, D(h)]=0$ we have $\{x \in \mathfrak{m}: S x=\lambda x\}$ is a non-zero $D(h)$ invariant subspace and must equal $\mathfrak{m}$. Thus $S=\lambda I$ and $\alpha(X, Y)=\frac{1}{2} X Y$. Thus any metric connection on $G / H$ given by fixed decomposition $\mathfrak{g}=\mathfrak{m}$ $+\mathfrak{h}$ and $a d_{\mathfrak{m}} \mathfrak{h}$ irreducible on $\mathfrak{m}$ must be an irreducible connection of the first kind. For complete details in this case see [16].

The above result has been generalized in sections 4.6 and 5.1 of [5]. For example, let $G / H$ be a Riemannian reductive homogeneous space such that $\mathfrak{g}=\mathfrak{m} \dot{+} \mathfrak{h}$ is simple. If $D(\mathfrak{h})$ has inequivalent representations in $\mathfrak{m}$, then $G / H$ is irreducible in any metric connection. " $D(\mathfrak{h})$ has inequivalent representations in 'm" means every irreducible representation of $D(\mathfrak{h})$ has at most multiplicity 1 on $\mathfrak{m}$; see [5]. We shall now use the algebra $(\mathfrak{m}, \alpha)$ to prove a variation of this result and to show the hypothesis is necessary.

Lemma 3. Let $G / H$ be a reductive homogeneous space with corresponding reductive pair $(\mathfrak{g}, \mathfrak{h})$ and decomposition $\mathfrak{g}=\mathfrak{m}+\mathfrak{h}$ satisfying

(a) $\mathfrak{g}=\mathfrak{m}+\mathfrak{h}(\mathfrak{m}, \mathfrak{m}) \quad(=\mathfrak{m}+[\mathfrak{m} \mathfrak{m}])$,

(b) $D(h)=0 \quad$ implies $h=0$,

(c) $G / H$ is completely reducible relative to some connection; i.e. there is an algebra $(\mathfrak{m}, \alpha)$ such that the corresponding holonomy algebra acts as a completely reducible Lie algebra on $\mathrm{m}$.

Then

(1) $\mathfrak{h}$ is reductive in $\mathfrak{g}$; i.e. ad $\mathfrak{g} \mathfrak{h}$ is completely reducible in $\mathfrak{g}$;

(2) $D(\mathfrak{h})$ is completely reducible in $\mathfrak{m}$.

Proof. Let $a(\mathfrak{m})$ be the Lie algebra generated by the endomorphisms $a(X): \mathfrak{m} \rightarrow \mathfrak{m}: Y \rightarrow \alpha(X, Y)$. Thus if $M_{1}=\{a(X): X \in \mathfrak{m}\}$ and $M_{k+1}=\left[M_{1}, M_{k}\right]$ we have $a(\mathfrak{m})=M_{1}+M_{2}+\cdots$; see [14]. From this and the fact $D(\mathfrak{h})$ consists of derivations of $(\mathfrak{m}, \alpha)$ we have $[D, a(X)]=a(D X)$ and by induction 
conclude $[D(\mathfrak{h}), a(\mathfrak{m})] \subset a(\mathfrak{m})$. Thus we see that $a(\mathfrak{m})+D(\mathfrak{h})$ is a Lie algebra and since the holonomy algebra, hol $(\alpha)$, is generated by $a(\mathfrak{m})$ and $D(\mathfrak{h})$ we have hol $(\alpha)=a(\mathfrak{m})+D(\mathfrak{h})$. Now by $(c)$ hol $(\alpha)$ is completely reducible and just as in the proof of Theorem 9 of [4] we can conclude that $D(\mathfrak{h})$ is completely reducible in $\mathfrak{m}$. Thus $D(\mathfrak{h})=c(D) \oplus D^{\prime}$ where $D^{\prime}$ is a semisimple ideal and the center of $D(\mathfrak{h})$ is $c(D)$ which consists of semi-simple endomorphisms.

Next we shall show that the map

$$
\phi: D(\mathfrak{h}) \rightarrow a d_{\mathfrak{h}} \mathfrak{h}: \quad D(h) \rightarrow a d_{\mathfrak{h}} h
$$

is an epimorphism of Lie algebras. First $D(h)=D(k)$ implies $D(h-k)=0$ and therefore by $(b), h=k$; thus the map $\phi$ is well defined. Next any $a d_{\mathfrak{h}} h$ determines an $h \in \mathfrak{h}$ so that $\phi: D(h) \rightarrow a d_{\mathfrak{h}} h$; thus $\phi$ is surjective. Finally using $[D(h), D(k)]=D\left(\left[\begin{array}{ll}h & k\end{array}\right]\right)$ we see that $\phi$ is an epimorphism of Lie algebras; thus $a d_{\mathfrak{h}} \mathfrak{h}=\operatorname{Im}(\phi)=\phi(D(\mathfrak{h}))$.

Next note that $\operatorname{Ker} \phi=c(D)$. For $0=\phi(D(h))=a d_{\mathfrak{\xi}} h$ yields for any $k \in \mathfrak{h}, \quad 0=\left(a d_{\mathfrak{h}} h\right)(k)=\left[\begin{array}{ll}h & k\end{array}\right]$. Thus $0=D\left(\left[\begin{array}{ll}h & k\end{array}\right]\right)=[D(h), D(k)]$ so that $D(h)$ $\in c(D)$. Conversely if $D(h) \in c(D)$, then for any $k \in \mathfrak{h}, 0=[D(h), D(k)]$ $=D\left(\left[\begin{array}{ll}h & k\end{array}\right]\right)$. Thus using $(b), 0=\left[\begin{array}{ll}h & k\end{array}\right]=\left(a d_{\mathfrak{\xi}} h\right)(k)$ so that $0=a d_{\mathfrak{h}} h=\phi(D(h))$. Using this we obtain

$$
a d_{\mathfrak{h}} \mathfrak{h}=\phi(D(\mathfrak{h}))=\phi(c(D)) \oplus \phi\left(D^{\prime}\right)=\phi\left(D^{\prime}\right),
$$

thus $a d_{\mathfrak{h}} \mathfrak{h}$ is semi-simple.

Next from $\mathfrak{g}=\mathfrak{m}+\mathfrak{h}$ we choose a basis of $\mathfrak{g}$ from $\mathfrak{m}$ and $\mathfrak{h}$ to obtain:

$$
a d_{\mathfrak{g}} \mathfrak{h}=\left[\begin{array}{cc}
D(\mathfrak{h}) & 0 \\
0 & a d_{\mathfrak{h}} \mathfrak{h}
\end{array}\right]=\left[\begin{array}{cc}
c(D) & 0 \\
0 & 0
\end{array}\right] \oplus\left[\begin{array}{cc}
D^{\prime} & 0 \\
0 & a d_{\mathfrak{h}} \mathfrak{h}
\end{array}\right]=c\left(a d_{\mathfrak{g}} \mathfrak{h}\right) \oplus\left(a d_{\mathfrak{g}} \mathfrak{h}\right)^{\prime}
$$

where

$$
c\left(a d_{\mathfrak{g}} \mathfrak{h}\right)=\left[\begin{array}{cc}
c(D) & 0 \\
0 & 0
\end{array}\right]
$$

is the center of $a d_{\mathfrak{g}} \mathfrak{h}$ and consists of semi-simple linear transformations and

$$
\left(a d_{\mathfrak{g}} \mathfrak{h}\right)^{\prime}=\left[\begin{array}{cc}
D^{\prime} & 0 \\
0 & a d_{\mathfrak{h}} \mathfrak{h}
\end{array}\right]
$$


is a semi-simple ideal. Thus by $[2, \mathrm{p} .81] a d_{g} \mathfrak{h}$ is completely reducible in g.

Remark. If $G$ is simple and $G / H$ is a Riemannian reductive nonsymmetric homogeneous space, then (a), (b), and (c) are satisfied: (a) since $\mathfrak{m}+\mathfrak{h}(\mathfrak{m}, \mathfrak{m})$ is a non-zero ideal of $\mathfrak{g}$; (c) using results in $[5 ; 10]$ on the connection of the first kind; and (b) as follows. Suppose $D(h)=0$, then

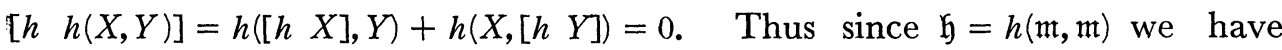
$a d_{\mathfrak{f}} h=0$. Therefore $a d_{\mathrm{g}} h=0$ so that $\left[\begin{array}{ll}h & g\end{array}\right]=0$. But this means $R h$ is a one dimensional ideal in $\mathfrak{g}$, a contradiction unless $h=0$.

Let $\mathfrak{g}=\mathfrak{m}+\mathfrak{h}$ be (fixed) decomposition which induces a metric connection of the first kind with metric $B$. Let $C(X, Y)=B(S X, Y)$ be another metric which determines another connection via the algebra $(\mathfrak{m}, \alpha)$ as previously discussed and let $\operatorname{hol}(B)$ and $\operatorname{hol}(C) \equiv \operatorname{hol}(\alpha)$ be the corresponding holonomy algebras, then we have the following result [5, Lemma 4. $4 \mathrm{~A}]$.

Lemma 4. Let $\mathfrak{n}$ be a subspace of $\mathfrak{m}$ such that $S \mathfrak{i n} \subset \mathfrak{i t}$ and $h o l(C) \mathfrak{n} \subset \mathfrak{n}$. Then $\operatorname{hol}(B) \mathfrak{n} \subset \mathfrak{n}$.

The proof can be given by means of the algebras ( $\mathfrak{m}, X Y)$ and $(\mathfrak{m}, \alpha)$ but we omit it.

Theorem 5. Let $G / H$ be a reductive homogeneous space with corresponding reductive pair $(\mathfrak{g}, \mathfrak{h})$ and decomposition $\mathfrak{g}=\mathfrak{m}+\mathfrak{h}$ satisfying

(a) $\mathfrak{g}=\mathfrak{m} \dot{+} \mathfrak{h}(\mathfrak{m}, \mathfrak{m})$,

(b) $D(h)=0$ implies $h=0$,

(c) $G / H$ is irreducible non-symmetric relative to a metric connection of the first kind (relative to above (fixed) decomposition),

(d) $D(\mathfrak{h})$ has inequivalent representations in $\mathfrak{m}$.

Then $G / H$ is irreducible relative to any metric connection.

Proof. Suppose not, then there exists a metric $C$ and a corresponding algebra $(\mathfrak{m}, \alpha)$ which has a $D(\mathfrak{h})$-invariant left ideal $\mathfrak{n}$; i.e. $\operatorname{hol}(C) \mathfrak{t} \subset \mathfrak{n}$. Now let $C(X, Y)=B(S X, Y)$ where $B$ induces the irreducible connection of the first kind, then $[S, D(\mathfrak{h})]=0$, i.e. $S \in$ centralizer $D(\mathfrak{h}) . \quad$ Since $D(\mathfrak{h}) \mathfrak{t} \subset \mathfrak{n}$ and $D(\mathfrak{h})$ is completely reducible, we may write - using $(d)-\mathfrak{n}=\mathfrak{m}_{1}+\cdots+\mathfrak{m}_{s}$. 
where the $\mathfrak{m}_{j}$ are $D(\mathfrak{h})$-irreducible and non-isomorphic $D(\mathfrak{h})$-modules. These $\mathfrak{m}_{j}{ }^{\prime} \mathrm{s}$ are actually homogeneous components of $D(\mathfrak{h})$ in $\mathfrak{m}$ and since $S$ $\in$ centralizer $D(\mathfrak{h})$ we have $S \mathfrak{m}_{j} \subset \mathfrak{m}_{j}$. Thus $S \mathfrak{t} \subset \mathfrak{t}$ and by Lemma $4 \mathrm{G} / \mathrm{H}$ is not irreducible relative to the connection of the first kind, a contradiction. (Perhaps see [3, p. 126] for homogeneous components using the associative enveloping algebra $\left.D^{*}\right)$.

Corollary 6. Let $G / H$ be a non-symmetric Riemannian reductive homogeneous space with $G$ simple and $\mathfrak{g}=\mathfrak{m}+\mathfrak{h}$ the corresponding decomposition. If $D(\mathfrak{G})$ has inequivalent representations in $\mathfrak{m}$, then $G / H$ is irreducible relative to any metric.

The proof uses Theorem 5, the remarks following Theorem 4, and results in [10] which show that $G / H$ is irreducible relative to the connection of the first kind.

The converse of this corollary appears to be an open problem. However, we now give an example to show the hypothesis on $D(\mathfrak{h})$ is necessary. Let $\mathrm{g}$ be the simple compact Lie algebra of $5 \times 5$ skew-symmetric matrices. Let $\mathfrak{h}$ be the simple Lie subalgebra of $3 \times 3$ skew-symmetric matrices imbedded in $g$ by:

$$
\mathfrak{h}=\left\{\left[\begin{array}{cc}
A & 0 \\
0 & 0
\end{array}\right]: A \text { is } 3 \times 3 \text { skew-symmetric }\right\} .
$$

Let $\mathfrak{m}=\mathfrak{h} \perp$ relative to the Killing form $B$ on $\mathfrak{g}$, then $\mathfrak{m}$ is given by:

$$
\mathfrak{m}=\left\{\left[\begin{array}{cc}
0 & X \\
-X^{\prime} & Y
\end{array}\right]: Y \text { is } 2 \times 2 \text { skew-symmetric }\right\} .
$$

Thus $(\mathfrak{g}, \mathfrak{h})$ is a reductive pair with $\mathfrak{g}=\mathfrak{m}+\mathfrak{h}$ and $-B \mid \mathfrak{m} \times \mathfrak{m}$ is positive definite and induces an irreducible metric connection of the first kind on the corresponding $\mathrm{G} / \mathrm{H}$.

Let $e_{i j}$ denote the usual matrix basis, then a basis for $\mathfrak{m}$ is

$$
\begin{aligned}
& f_{1}=e_{14}-e_{41}, \quad f_{2}=e_{24}-e_{42}, \quad f_{3}=e_{34}-e_{43} ; \\
& f_{1}^{\prime}=e_{15}-e_{51}, \quad f_{2}^{\prime}=e_{25}-e_{52}, \quad f_{3}^{\prime}=e_{35}-e_{53} ; \text { and } \\
& f_{4}=e_{45}-e_{54} .
\end{aligned}
$$

Let $\mathfrak{m}_{1}$ (resp. $\mathfrak{m}_{2}$ ) be the subspace spanned by the $f_{i}^{\prime}$ 's (resp. $f_{i}^{\prime}$ 's) and let $\mathfrak{m}_{3}=R f_{4}$. These subspaces are $D(\mathfrak{h})$-submodules and $\mathfrak{m}_{1} \rightarrow \mathfrak{m}_{2}: f_{i} \rightarrow f_{i}^{\prime}$ yields a $D(\mathfrak{h})$-module isomorphism; also $D(\mathfrak{h}) \mathfrak{m}_{3}=0$. 
Next we note that the algebra $(\mathfrak{m}, X Y)$ is given by:

$$
\begin{aligned}
& \mathfrak{m}_{i}{ }^{2}=0 \quad i=1,2,3 ; \\
& f_{4} f_{i}=-f_{i}^{\prime}, \quad f_{4} f_{i}^{\prime}=f_{\imath}, \quad f_{i} f_{j}^{\prime}=-\delta_{i j} f_{4} .
\end{aligned}
$$

Let $I$ denote the $3 \times 3$ identity matrix and let

$$
S=\left[\begin{array}{lll}
p I & & 0 \\
& q I & \\
0 & & r
\end{array}\right]
$$

be the endomorphism on $\mathfrak{m}=\mathfrak{m}_{1}+\mathfrak{m}_{2}+\mathfrak{m}_{3}$ which defines the metric $C(X, Y)$ $=-B(S X, Y)$; we assume

$$
p+q-r=0 \text { and } \operatorname{det}(S)>0 .
$$

We next have that the algebra $(\mathfrak{m}, \alpha)$ which induces the $C$-metric connection is given by:

$$
\begin{aligned}
& \alpha\left(\mathfrak{m}_{i}, \mathfrak{m}_{i}\right)=0 \quad i=1,2,3 ; \\
& 2 \alpha\left(f_{i}, f_{j}^{\prime}\right)=\frac{1}{r} \delta_{i j}(r+q-p) f_{4} ; \\
& \alpha\left(f_{4}, f_{i}\right)=\alpha\left(f_{4}, f_{i}^{\prime}\right)=0 .
\end{aligned}
$$

Let $\mathfrak{i t}$ be the 4-dimensional subspace spanned by $f_{4}$ and $f_{i}+f_{i}^{\prime}$ with $i=1,2,3$. Then since $\mathfrak{m}_{1} \cong \mathfrak{m}_{2}$ as above and $D(h) f_{4}=0$, we see that $\mathfrak{n}$ is $D(\mathfrak{h})$-invariant. Also using the above multiplication and $\alpha(X, Y)=X Y$

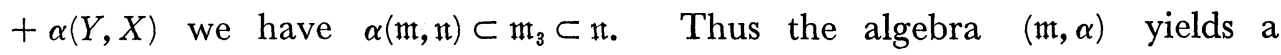
reducible connection on the corresponding $G / H$.

We now change $S$ slightly and obtain a new algebra $(\mathfrak{m}, \alpha)$ which gives an irreducible metric connection. Assume that

$$
a=p+q-r \neq 0, \quad b=p-q-r \neq 0, \quad \text { and } \operatorname{det}(S)>0 .
$$

Then we obtain an algebra $(\mathfrak{m}, \alpha)$ with multiplication

(a) $\alpha\left(\mathfrak{m}_{i}, \mathfrak{m}_{\imath}\right)=0 \quad i=1,2,3$;

(b) $\alpha\left(f_{i}, f_{j}^{\prime}\right)=-\delta_{i j} \frac{b}{r} f_{4}$,

(c) $2 \alpha\left(f_{4}, f_{i}\right)=\frac{-a}{q} f_{i}^{\prime}, 2 \alpha\left(f_{4}, f_{i}^{\prime}\right)=\frac{a}{p} f_{1}$.

Now if $a_{i} \in \mathfrak{m}_{i}$ are such that $a=a_{1}+a_{2}+a_{3} \in \mathfrak{n}$ which is a $D(\mathfrak{h})$ invariant left ideal of $(\mathfrak{m}, \alpha)$, then using the $D(\mathfrak{h})$-invariance, we easily obtain that elements of the form $u_{i} f_{i}+v_{i} f_{i}^{\prime}$ (with $u_{i}, v_{i} \in R$ ) are in $\mathfrak{n}$ for $i=1,2,3$. 
Using this and (b) we see that $f_{4} \in \mathfrak{t}$. With $f_{4} \in \mathfrak{t t}$ we next use (c) and $\alpha(X, Y)=\alpha(Y, X)+X Y$ and $a, b \neq 0$ to obtain $f_{i}, f_{i}^{\prime} \in \mathfrak{H} ;$ thus $\mathfrak{i}=\mathfrak{m}$.

Next we give a less computational example of a reductive pair $(\mathfrak{g}, \mathfrak{h})$ with $g$ and $h$ simple but $g$ not compact and the corresponding $G / H$ is reducible in a naturally defined connection. Let $g=g \mid(n)$ be the Lie algebra of all $n \times n$ matrices under commutation and let $\mathfrak{h}$ be the subalgebra of $q \times q$ matrices of trace 0 imbedded as follows:

$$
\mathfrak{h}=\left\{\left[\begin{array}{rr}
0 & 0 \\
0 & U
\end{array}\right]: \operatorname{tr} U=0\right\} .
$$

Let $\mathfrak{m}$ be the subspace of $\mathfrak{g}$ spanned by

$$
\left[\begin{array}{ll}
A & B \\
C & 0
\end{array}\right] \text { and } I \text {, }
$$

then $\mathfrak{g}=\mathfrak{m}+\mathfrak{h}$ with $[\mathfrak{m}, \mathfrak{h}] \subset \mathfrak{m}$. Next for

$$
X=\left[\begin{array}{ll}
A & B \\
C & 0
\end{array}\right]+a I, \quad Y=\left[\begin{array}{ll}
P & Q \\
R & 0
\end{array}\right]+p I \in \mathfrak{m}
$$

we define

$$
\alpha(X, Y)=\left[\begin{array}{cc}
A P+B R & A Q \\
C P & 0
\end{array}\right]+\left(\frac{1}{n} \operatorname{tr} C Q\right) I .
$$

Then $(\mathfrak{m}, \alpha)$ is an algebra such that

$$
\begin{aligned}
& \alpha(X, Y)-\alpha(Y, X)=X Y \\
= & {\left[\begin{array}{cc}
{[A, P]+B R-Q C} & A Q-P B \\
C P-R A & 0
\end{array}\right]+\frac{1}{n} \operatorname{tr}(C Q-R B) I }
\end{aligned}
$$

and

$$
D \alpha(X, Y)=\alpha(D X, Y)+\alpha(X, D Y)
$$

using

$$
D(h) X=\left[\begin{array}{cc}
0 & -B U \\
U C & 0
\end{array}\right]
$$

where $D=D(h)$ with $h \in \mathfrak{h}$ as above. Thus (m, $\alpha$ ) defined a connection on the corresponding $G / H$ with zero torsion. 
This connection is reducible since we can construct left ideals of ( $\mathfrak{m}, \alpha)$ analogous to those in the associative matrix algebra. For let $P$ and $R$ be matrices with 0 's in the first column, then the set of elements $\mathfrak{n}$ given by:

$$
\mathfrak{H}=\left\{\left[\begin{array}{ll}
P & 0 \\
R & 0
\end{array}\right]: P, R \text { as above }\right\}
$$

is $D(\mathfrak{h})$-invariant left ideal of $(\mathfrak{m}, \alpha)$.

Next since the ideal $\mathfrak{t}=R I$ of the Lie algebra $g$ is $\mathfrak{h}$-invariant, the Lie algebra homomorphism $g \rightarrow \bar{g}=g / \mathfrak{f}$ yields a reductive pair $(\overline{\mathfrak{g}}, \overline{\mathfrak{h}})$ with $\overline{\mathfrak{g}}=\overline{\mathfrak{m}}+\overline{\mathfrak{h}}$ where $\overline{\mathfrak{h}}$ is isomorphic to $\mathfrak{h}$ and $[\overline{\mathfrak{m}} \overline{\mathfrak{h}}]=[\overline{\mathfrak{m} \mathfrak{h}}] \subset \overline{\mathfrak{m}}$; thus we have the reductive pair $(\overline{\mathfrak{g}}, \overline{\mathfrak{h}})$ with $\overline{\mathfrak{g}}$ and $\overline{\mathfrak{h}}$ simple and $\overline{\mathfrak{g}}$ not compact. Also $(\overline{\mathfrak{m}}, \bar{X} \bar{Y})$ is a homomorphic image of the algebra (m, $X Y)$ with $\bar{X} \bar{Y}=\overline{X Y}$ and $D(\bar{h}) \bar{X}=\overline{[h, X}]$. Now on $\overline{\mathfrak{m}}$ define the multiplication $\bar{\alpha}$ by

$$
\bar{\alpha}(\bar{X}, \bar{Y})=\overline{\alpha(X, Y)} .
$$

By the construction of $\alpha$ we see that $\bar{\alpha}$ is well defined and satisfies the following: $\bar{\alpha}(\bar{X}, \bar{Y})-\bar{\alpha}(\bar{X}, \bar{Y})=\bar{X} \bar{Y}$ in $\overline{\mathfrak{m}} ; D(\bar{h})$ is a derivation of $(\overline{\mathfrak{m}}, \bar{\alpha}) ;$ and $\overline{\mathfrak{m}}=\overline{\mathfrak{h}}^{\perp}$ relative to the Killing form $\bar{B}$ of $\overline{\mathfrak{g}}$. Furthermore $\bar{B} \mid \overline{\mathfrak{m}} \times \overline{\mathfrak{m}}$ induces a non-degenerate invariant form $\bar{C}$ on the algebra $(\overline{\mathfrak{m}}, \bar{\alpha})$; thus $(\overline{\mathfrak{m}}, \bar{\alpha})$ induces a reducible pseudo-Riemannian connection on the corresponding $\bar{G} / \bar{H}$.

As a final application of the algebra $(\mathfrak{m}, \alpha)$ we use the results in [10, section 4] which relate affine transformation of $G / H$ and algebra automorphisms of $(\mathfrak{m}, \alpha)$.

TheOREm. Let $G / H$ be a reductive homogeneous space with corresponding reductive pair $(\mathfrak{g}, \mathfrak{h})$ and fixed decomposition $\mathfrak{g}=\mathfrak{m}+\mathfrak{h}$. Let $(\mathfrak{m}, \alpha)$ be an algebra which induces a connection on $G / H$. Let $\phi: G / H \rightarrow G / H$ be an analytic diffeomorphism which locally commutes with $G$ and $\phi(H)=H$. Then $\phi$ is an affine transformation on $G / H$ if and only if $\phi^{\prime}$ is an automorphism of the algebra ( $\left.\mathfrak{m}, \alpha\right)$ where $\phi^{\prime}$ is the differential of $\phi$ at the point $H \in G / H$.

To apply this let $\Sigma$ be an analytic automorphism of a semi-simple Lie group $G$. Let $\sigma$ be the corresponding automorphism of $\mathfrak{g}$ and let $\mathfrak{h}$ (resp. $\mathfrak{m}$ ) be the Fitting zero (resp. one) component of $g$ relative to $\sigma-I$; see [2, p. 37]. Thus $\mathfrak{h}=\left\{z \in g:(\sigma-I)^{k} z=0\right.$, some $\left.k\right\}$ is a subalgebra of $\mathrm{g}$ and $\sigma-I$ is non-singular on $\mathfrak{m}$ and $\mathfrak{g}=\mathfrak{m}+\mathfrak{h}$ (analogous to [2, p. 54, ex. 5]). Using $\sigma$ is an automorphism and $B(\sigma P, \sigma Q)=B(P, Q)$ where $B$ is the 
Killing form of $\mathfrak{g}$, we obtain $\mathfrak{m}=\mathfrak{h} \perp$ relative to $B$ and $[\mathfrak{m} \mathfrak{h}] \subset \mathfrak{m}$. Thus $(\mathfrak{g}, \mathfrak{h})$ is a reductive pair and if $H$ is the subgroup of $G$ corresponding to $\mathfrak{h}$, we see $G / H$ is a reductive space such that $B \mid \mathfrak{m} \times \mathfrak{m}$ induces a pseudoRiemannian connection of the first kind on $G / H$; note [13, section 4].

Since $H$ is $\Sigma$-invariant, $\Sigma$ induces a diffeomorphism $\phi: G / H \rightarrow G / H$ : $a H \rightarrow \sum a H$ which locally commutes with $G$ (because $\Sigma$ is an automorphism). Thus the algebra $(\mathfrak{m}, X Y)$ has $\phi^{\prime}$ as an automorphism using $\phi^{\prime}=\sigma \mid \mathfrak{m}$. Other algebras $(\mathfrak{m}, \alpha)$ such that $\phi^{\prime}$ is an automorphism (and consequently $\phi$ is an affine transformation) are as follows. Let $S=S^{b} \in \operatorname{Hom}(\mathfrak{m}, \mathfrak{m})$ satisfy $\left[S, \phi^{\prime}\right]$ $=0$ e.g. $S$ is a suitable polynomial in $\phi^{\prime}$, and define $\alpha(X, Y)$ by formula (10). We use $\phi^{\prime}$ is an automorphism of ( $\left.\mathfrak{m}, X Y\right)$ to obtain $\phi^{\prime} L(X) \phi^{\prime-1}$ $=L\left(\phi^{\prime} X\right)$ and use this with $\left[\phi^{\prime}, S\right]=0$ in (10) to obtain $\phi^{\prime} a(X) \phi^{\prime-1}=a\left(\phi^{\prime} X\right)$. Thus $\phi^{\prime}$ is an automorphism of $(\mathfrak{m}, \alpha)$.

Note that in general if we let $C(X, Y)=B(S X, Y)$ where $B, \phi^{\prime}, \mathfrak{m}$ and $\mathfrak{h}$ are as above and require $C\left(\phi^{\prime} X, \phi^{\prime} Y\right)=C(X, Y)$, then using $B\left(\phi^{\prime} X, \phi^{\prime} Y\right)$ $=B(X, Y)$ we obtain $\left[\phi^{\prime}, S\right]=0$. Thus $\phi^{\prime}$ is an automorphism of the corresponding algebra $(\mathfrak{m}, \alpha)$; i.e. $\phi$ is a $C$-isometry implies $\phi$ is a $C$-affine transformation.

4. Holonomy algebra. Let $(\mathfrak{g}, \mathfrak{h})$ be a reductive pair with $\mathfrak{g}=\mathfrak{m}+\mathfrak{h}$ a fixed decomposition. Let $a(X): \mathfrak{m} \rightarrow \mathfrak{m}: Y \rightarrow \alpha(X, Y)$ and let $b(X): \mathfrak{m} \rightarrow \mathfrak{m}$ : $Y \rightarrow \alpha(Y, X)$ be the right and left multiplications for the algebra (nt, $\alpha)$ which defines a connection on the corresponding $G / H$. Let $L(\alpha)$ be the Lie algebra generated by the $a(X)$ 's and $b(Y)$ 's for all $X, Y \in \mathfrak{m}$; see [14;10].

TheORem 7. Let $(\mathfrak{g}, \mathfrak{h})$ be a non-symmetric reductive pair such that the above algebra $(\mathfrak{m}, \alpha)$ defines an irreducible connection with zero torsion on the corresponding $G / H$. Then $h o l(\alpha)$ and $L(\alpha)$ are irreducible Lie algebras. If the connection is pseudo-Riemannian, then $\operatorname{hol}(\alpha) \subset L(\alpha)$; in this case $h o l(\alpha)=L(\alpha)$ if and only if $(\mathfrak{m}, \alpha)$ induces a connection of the first kind.

Proof. Since $G / H$ is irreducible we have hol $(\alpha)$ is irreducible and from Theorem $1(\mathfrak{m}, \alpha)$ is simple so that $L(\alpha)$ is also irreducible; (noting that an ideal of $(\mathfrak{m}, \alpha)$ is just an $L(\alpha)$-invariant subspace).

Next assume $(\mathfrak{m}, \alpha)$ induces a pseudo-Riemannian connection on $G / H$ via the nondegenerate form $C(X, Y)$ defined on $\mathfrak{m}$. Since $\operatorname{hol}(\alpha)=a(\mathfrak{m})+D(\mathfrak{h})$ it suffices to show $D(\mathfrak{h}) \subset L(\alpha)$ to prove hol $(\alpha) \subset L(\alpha)$; see [10] when the 
connection is of the first kind. Let $L^{*}$ be associative enveloping algebra of $L(\alpha)$; see [2]. Since $L(\alpha)$ is irreducible, $L^{*}$ is semi-simple and since any $D \in D(\mathfrak{h})$ is a derivation of $(\mathfrak{m}, \alpha),[D, L(\alpha)] \subset L(\alpha)$ and therefore $\left[D, L^{*}\right]$ $\subset L^{*}$. Thus $D$ induces a derivation $\bar{D}: L^{*} \rightarrow L^{*}: Q \rightarrow[D, Q]$. Since $L^{*}$ is a semi-simple associative algebra, its derivations are inner [14]. Thus there exists $U \in L^{*}$ such that $\bar{D} Q=[U, Q]$ for all $Q \in L^{*}$. Next since $L(\alpha)$ is irreducible, $L(\alpha)=c(L) \oplus L^{\prime}$ where $L^{\prime}$ is semi-simple and $c(L)$ is the center of $L(\alpha)$; see [2, p. 81]. Also since $D^{\prime}: L^{\prime} \rightarrow L^{\prime}: P \rightarrow[D, P]$ is a derivation of the semi-simple Lie algebra, there exists $V \in L^{\prime}$ with $D^{\prime} P=a d V P=[V, P]$ for all $P \in L^{\prime}$. Next we note that for all $C \in c(L) \subset L^{*},[D, C]=\bar{D} C$ $=[U, C]=0$, since $\left[C, L^{*}\right]=0$. Thus for any $R=C+P \in L(\alpha)$ we have $[D, R]=[D, P]=[V, P]=[V, R]$. Thus $[D-V, R]=0$ for all $R \in L(\alpha)$ and consequently $\quad D-V=T \in \Gamma(\mathfrak{m}, \alpha)=\{S \in \operatorname{Hom}(\mathfrak{m}, \mathfrak{m}):[S, a(X)]=[S, b(X)]=0$ all $X \in \mathfrak{m}\}$ which is the centroid of the algebra $(\mathfrak{m}, \alpha)$. Since $(\mathfrak{m}, \alpha)$ is simple, $\Gamma(\mathfrak{m}, \alpha)$ is a field [2, p. 291] and for any $S \in \Gamma(\mathfrak{m}, \alpha)$ it is easy to see $S a(X)$ $=a(S X) ; S b(X)=b(S X)$ and using this $S L(\alpha) \subset L(\alpha)$ and $S L^{\prime} \subset L^{\prime}$. Thus trace $S P=0$ for all $S \in \Gamma(\mathfrak{m}, \alpha)$ and $P \in L^{\prime}$.

Next since $C(X, Y)$ is nondegenerate form which induces a pseudoRiemannian connection we have all the $D(h)$ and $a(X)$ are $C$-skew-symmetric. We shall now show every $S \in \Gamma(\mathfrak{m}, \alpha)$ is $C$-symmetric.

$$
\begin{aligned}
C(S \alpha(X, Y), Z) & =C(S a(X) Y, Z) \\
& =C(a(S X) Y, Z) \\
& =-C(Y, a(S X) Z) \\
& =-C(Y, S a(X) Z) \\
& =-C(Y, a(X) S Z) \\
& =C(\alpha(X, Y), S Z)
\end{aligned}
$$

and since $(\mathfrak{m}, \alpha)$ is simple, $\mathfrak{m}=\alpha(\mathfrak{m}, \mathfrak{m})$ thus $C(S U, Z)=C(U, S Z)$ for any $U \in \mathfrak{m}$.

Using $D=V+T$ with $V \in L^{\prime}, T \in \Gamma(\mathfrak{m}, \alpha)$ we have for any $S \in \Gamma(\mathfrak{m}, \alpha)$ that $[D, S]=0$ (remembering $\Gamma(\mathfrak{m}, \alpha)$ is commutative).

Thus

$$
\begin{aligned}
C(D S U, Z) & =-C(S U, D Z) \\
& =-C(U, S D Z) \\
& =-C(U, D S Z)
\end{aligned}
$$

so that $\operatorname{tr} D S=0$. 
Now from above equation $D=V+T$ we see that if $T \neq 0$, then $T^{-1}$ $\in \Gamma(\mathfrak{m}, \alpha)$ and therefore $I=T^{-1} T=T^{-1} D-T^{-1} V$. Thus using the results of previous paragraphs with $S=T^{-1}$ we obtain the contradiction $\operatorname{tr} I=0$. Thus $D=V \in L^{\prime}$.

To prove the last part we note that if the connection is of the first kind, then $2 \alpha(X, Y)=X Y$ so that $b(X)=-a(X)$ and $L(\alpha) \subset \operatorname{hol}(\alpha)$. Thus $L(\alpha)=\operatorname{hol}(\alpha)$. Conversely we see that if $b(X) \in \operatorname{hol}(\alpha)=a(\mathfrak{m})+D(\mathfrak{h})$, then $b(X)=A(X)+D$ with $A(X) \in a(\mathfrak{m})$. Since torsion is zero, $b(X)=a(X)-L(X)$ where $L(X): \mathfrak{m} \rightarrow \mathfrak{m}: Y \rightarrow X Y$. Thus $L(X) \in \operatorname{hol}(\alpha)$ and is $C$-skew-symmetric. This yields $C(X Y, Z)=C(X, Y Z)$ and from (9) we obtain $2 \alpha(X, Y)=X Y$.

Corollary 8. Let $G / H$ be a reductive homogeneous space such that $G / H$ is an irreducible pseudo-Riemannian space as in Theorem 7. Then hol $(\alpha)$ is a reductive subalgebra of $L(\alpha)$ which is contained in $L^{\prime}$.

Proof. Let $L(\boldsymbol{\alpha})=c(L) \oplus L^{\prime} \supset \operatorname{hol}(\boldsymbol{\alpha})$ and let $V=C+V^{\prime} \in \operatorname{hol}(\boldsymbol{\alpha})$ where $C \in c(L), V^{\prime} \in L^{\prime}$. Thus $C \in \Gamma(\mathfrak{m}, \alpha)$ and if $C \neq 0$, let $S=C^{-1} \in \Gamma(\mathfrak{m}, \alpha)$. Then $S V=I+S V^{\prime}$ and from the preceding proof, $\operatorname{tr} S V^{\prime}=0$. Also since $S$ is $C$-symmetric and $V \in \operatorname{hol}(\alpha)$ is $C$-skew-symmetric, we have $\operatorname{tr} S V=0$. Thus the contradiction $\operatorname{tr} I=0$ shows $C=0$ and hol $(\alpha) \subset L^{\prime}$.

Next we shall show $a d_{L} \operatorname{hol}(\alpha)$ is completely reducible in $L=L(\alpha)$ by using [2, p. 81]. Since $\mathfrak{h}^{*} \equiv \mathrm{hol}(\alpha)$ is a subalgebra of $L(\alpha)$, the map $\mathfrak{h}^{*} \rightarrow a d_{L} \mathfrak{h}^{*}: A \rightarrow a d A$ is a representation which is faithful. For if $a d_{L} A=0$, then $[A, P]=0$ for all $P \in L^{\prime}$. But since $A \in \mathfrak{h}^{*} \subset L^{\prime}$ we see that $A$ is in the radical of $L^{\prime}$ which is zero.

From the holonomy irreducibility we have the usual decomposition $\mathfrak{h}^{*}=c\left(\mathfrak{h}^{*}\right) \oplus \mathfrak{h}^{* \prime}$ and consequently $a d_{L} \mathfrak{h}^{*}=a d c\left(\mathfrak{h}^{*}\right)+a d \mathfrak{h}^{* \prime}$. Since $a d \mid L^{\prime}$ is a faithful representation, this sum is direct; ad $\mathfrak{h}^{* \prime}$ is semi-simple; and $a d c\left(\mathfrak{h}^{*}\right)$ is the center of $a d_{L} \mathfrak{h}^{*}$. Thus to show $a d_{L} \mathfrak{h}^{*}$ completely reducible, we shall show that the elements in $a d c\left(\mathfrak{h}^{*}\right)$ are semi-simple linear transformations.

Now let $0 \neq A \in c\left(\mathfrak{h}^{*}\right)$, then since $\mathfrak{h}^{*}$ is irreducible $c\left(\mathfrak{h}^{*}\right)$ is contained in a division ring which is at worst isomorphic to the quaternions. In particular $A$ satisfies a quadratic equation $A^{2}+a A+b I=0$ with $a, b \in R$. Since $A \in \mathfrak{h}^{*}, A$ is $C$-skew and $\operatorname{tr} A=0$; thus $a=0$. Next $b>0$, otherwise $A^{2}+b I=0$ factors so that we may conclude $A=\lambda I$ and therefore, by traces, $A=0$. Thus $b=\beta^{2}>0$ and for $J=A / \beta \in c\left(h^{*}\right)$ we have $J^{2}+I=0$. Using 
this a straight forward computation shows that $a d_{L} J$ satisfies a polynomial $x\left(x^{2}+4\right) \in R[x]$; thus the minimum polynomial of $a d_{L} J$ is a product of distinct irreducible polynomials so that the linear transformations in ad $c\left(\mathfrak{h}^{*}\right)$ are semi-simple.

Note that in general $\operatorname{hol}(\alpha)$ is not an ideal of $L(\alpha)$; e.g. $L(\alpha)$ semisimple but hol $(\alpha)=\mathfrak{h}^{*}$ such that $c\left(\mathfrak{h}^{*}\right) \neq 0$.

We have somewhat determined the location of $\mathfrak{h}^{*}=\operatorname{hol}(\alpha)$ in $L(\alpha)$ and we next consider the semi-simplicity of these algebras.

THEOREM 9. Let $(\mathfrak{g}, \mathfrak{h})$ be a non-symmetric reductive pair with $\mathfrak{g}=\mathfrak{m}+\mathfrak{h}$ such that the algebra $(\mathfrak{m}, \alpha)$ induces an irreducible pseudo-Riemannian connection on the corresponding $G / H$. Then

(1) If $\mathfrak{g}$ is semi-simple, then $L(\alpha)$ is semi-simple.

(2) If $c\left(\mathfrak{h}^{*}\right) \neq 0$, then $c\left(\mathfrak{h}^{*}\right)=R J$ where $J^{2}+I=0$ and $J$ induces a $G$-invariant pseudo-Kaehlerian structure on $G / H$. Conversely if $G / H$ has a G-invariant pseudoKaehlerian structure $\mathfrak{s}$ and if $c\left(\mathfrak{h}^{*}\right) \neq 0$, then $J=\mathfrak{s}(p) \in c\left(h^{*}\right)$ where $p=H \in G / H$.

(3) If dim. $\mathfrak{m}$ is odd, then $\operatorname{hol}(\alpha)$ is semi-simple.

(4) If $G / H$ is Riemannian and $c(L) \neq 0$, then $c(L)=R I$.

(5) If $c(L) \neq 0$ and $c\left(\mathfrak{h}^{*}\right) \neq 0$, then $c(L)=R I$ and $c\left(\mathfrak{h}^{*}\right)=R J$ with $J^{2}+I$ $=0$.

(6) If $G / H$ has a G-invariant quaternionic structure, then the connection is not of the first kind.

Proof. (1) Choosing a basis for $\mathfrak{g}$ from $\mathfrak{m}$ and $\mathfrak{h}$ we obtain for $X \in \mathfrak{m}$ that

$$
\operatorname{ad}_{\mathrm{g}} X=\left[\begin{array}{cc}
L(X) & * \\
* & 0
\end{array}\right]
$$

where $L(X): \mathfrak{m} \rightarrow \mathfrak{n}: Y \rightarrow X Y$. Thus since $\mathfrak{g}$ is semi-simple, $0=\operatorname{tr} a d X$ $=\operatorname{tr} L(X)$. Next since the connection is pseudo-Riemannian we also have $\operatorname{tr} a(X)=0$. But $a(X)=b(X)-L(X)$ yields $\operatorname{tr} b(X)=0$ and since $L(\alpha)$ is generated by $a(\mathfrak{m})+b(\mathfrak{m})$ we see $\operatorname{tr} T=0$ all $T \in L(\alpha)$.

Since the connection is irreducible $(\mathfrak{m}, \alpha)$ is simple and $L(\alpha)=c(L) \oplus L^{\prime}$ is irreducible where $c(L) \subset \Gamma(\mathfrak{m}, \alpha)$ is a field. If $0 \neq A \in c(L)$, then $A^{-1}$ $\in \Gamma(\mathfrak{m}, \alpha)$ and $I=A^{-1} A \in \Gamma(\mathfrak{m}, \alpha) L(\alpha) \subset L(\alpha)$ must have trace zero, a contradiction. Thus $c(L)=0$ so that $L(\alpha)$ is semi-simple. 
(2) Assume $c\left(\mathfrak{h}^{*}\right) \neq 0$ and let $A, B \in c\left(\mathfrak{h}^{*}\right)$ be linearly independent. Now since $c\left(\mathfrak{h}^{*}\right) \subset$ centralizer $\left(\mathfrak{h}^{*}\right)$ which is a division algebra over $R$; see [2], we have that the subdivision algebra $K=R(A, B)$ generated by $A$ and $B$ is actually a field, using $[A, B]=0$. Thus $K$ is a field which is a proper finite extension of $R$ and is isomorphic to the complex numbers; thus dim. $K=2$ and $K=R A+R B \subset c\left(\mathfrak{h}^{*}\right)$. But $A^{-1} \in K$ and $I=A^{-1} A \in K$ $\subset c\left(\mathfrak{G}^{*}\right)$ so that $\operatorname{tr} I=0$. This contradiction shows $\operatorname{dim} . c\left(\mathfrak{h}^{*}\right)=1$.

Let $R A=c\left(\mathfrak{G}^{*}\right) \subset$ centralizer $\left(\mathfrak{G}^{*}\right)$ which is at worst isomorphic to the quaternions so that $A$ is quadratic: $A^{2}+a A+b I=0$. But $\operatorname{tr} A=0$ yields $a=0$; also $b>0$, otherwise $A= \pm b^{1 / 2} I$. Thus for $J=b^{-1 / 2} A$ we have $J^{2}+I=0$ and $c\left(\mathfrak{h}^{*}\right)=R J$. Now since $J$ commutes with $\mathfrak{h}^{*}=\operatorname{hol}(\alpha)$ and $J$ is $C$-skew where $C(X, Y)$ induces the pseudo-Riemannian structure, we have $C(J X, J Y)=C(X, Y)$. Thus analogous to [1, Prop 4. 2, p. 302], $J$ induces a pseudo-Kaehlerian $\mathfrak{s}$ on $G / H$. Conversely using the results of $[18$, p. 87] we see that the pseudo-Kaehlerian structure $\mathfrak{s}$ on $G / H$ yields $\Im(p)=A \in$ centralizer $\left(\mathfrak{G}^{*}\right)$ such that $A$ is $C$-skew and therefore $\operatorname{tr} A=0$. But $c\left(\mathfrak{h}^{*}\right)=R J$ so that $[A, J]=0$ and as above $K=R(A, J)$ is a 2-dimensional field if $A$ and $J$ are linearly independent. But this leads to $\operatorname{tr} I=0$; thus $A=a J \in c\left(\mathfrak{h}^{*}\right)$.

(3) If $\operatorname{dim} . \mathfrak{m}$ is odd, then $c\left(\mathfrak{h}^{*}\right)=0$; otherwise $(\operatorname{det} J)^{2}=\operatorname{det}\left(J^{2}\right)$ $=\operatorname{det}(-I)=-1$.

(4) If $c(L) \neq 0$ and $c(L) \neq R I$, then the above proofs show $c(L)=\Gamma(\mathfrak{m}, \alpha)$ $=R I+R J$ where $J^{2}+I=0$. Now as in the proof of Theorem $7, J$ is $C$-symmetric and since $C$ is positive definite (and therefore $J$ has a symmetric matrix), $J$ has real characteristic roots. This contradiction shows $c(L)=R I$.

(5) If $c\left(\mathfrak{h}^{*}\right) \neq 0$, then $c\left(\mathfrak{G}^{*}\right)=R J$. If $c(L) \neq R I$, then $c(L)=R I \oplus R P$ with $P^{2}+I=0$. From Corollary $8, c\left(\mathfrak{h}^{*}\right) \subset L^{\prime}$ and therefore $[P, J]=0$. Thus since the independent elements $I, J, P \in K=R(J, P)$ we see that $K$ is a 3-dimensional subfield of centralizer $\left(\mathfrak{h}^{*}\right)$ which is isomorphic to the quaternions; this is impossible.

(6) Let $G / H$ be a pseudo-Riemannian space with a quaternionic structure [7, 15] which is compatible with the connection of the first kind. The compatibility yields (analogous to [18]) a set $Q=\{I, J, K, L\}$ of endo- 
morphisms which relative to the usual endomorphism multiplication is isomorphic to the quaternions and such that $\left[Q, \mathfrak{h}^{*}\right]=0$. Thus $Q \subset$ centralizer $\left(\mathfrak{b}^{*}\right)$. But since we are assuming the connection to be of the first kind, $L(\alpha)=\mathfrak{h}^{*}=\operatorname{hol}(\alpha)$ so that the centralizer $\left(\mathfrak{h}^{*}\right)=\Gamma(\mathfrak{m}, X Y)$ is a field containing $Q$ which is impossible.

We now use preceding results to reduce the study of an irreducible pseudo-Riemannian connection on $G / H$ induced by the algebra $(\mathfrak{m}, \alpha)$ to the study of a finite family of anti-commutative algebras $M_{i}$ which yield reductive spaces $\mathscr{L}_{i} / \mathscr{K}_{i}$ which are irreducible symmetric or irreducible with connection of the first kind.

THEOREM 10. Let $(\mathfrak{g}, \mathfrak{h})$ be a non-symmetric reductive pair with fixed decomposition $\mathfrak{g}=\mathfrak{m}+\mathfrak{h}$ such that $\mathfrak{g}$ is semi-simple and the algebra ( $\mathfrak{m}, \alpha)$ induces an irreducible pseudo-Riemannian connection on the corresponding $G / H$. Then the Lie algebra $L=L(\alpha)$ and the reductive subalgebra $\mathfrak{h}^{*}=h o l(\alpha)$ are such that

(a) $\left(L, \mathfrak{h}^{*}\right)$ is a reductive pair with $L$ semi-simple and has decomposition $L=M+\mathfrak{h}^{*}$ where $M=\mathfrak{h}^{* \perp}$ relative to the Killing form, $K$, of $L$.

(b) $L=L_{1} \oplus \cdots \oplus L_{k}$ where each $L_{\imath}$ is a semi-simple ideal of $L . \quad$ Each $L_{\imath}$ contains a reductive subalgebra $\mathfrak{h}_{i}^{*}$ which is an ideal of $\mathfrak{h}^{*}$ such that $\left(L_{\imath}, \mathfrak{h}_{i}^{*}\right)$ is a reductive pair with decomposition $L_{i}=M_{i} \dot{+} \mathfrak{h}_{i}^{*}$ where $M_{i}=\mathfrak{h}_{i}^{*} \mathcal{\perp}$ relative to $K_{i}=K \mid L_{i} \times L_{i}$. Furthermore with the usual anti-commutative multiplication induced from $L_{i}$, the $M_{i}$ is a zero algebra or a simple algebra. Examples show that in general the case $L_{i}$ semi-simple can not be reduced to $L_{i}$ simple.

Proof. The proofs are analogous to those in $[11$, section $2 ; 13$, section 3] and are outlined below. We first show the Killing form $K$ of $L$ restricted to $\mathfrak{h}^{*} \times \mathfrak{h}^{*}$ is nondegenerate. The representation $\mathfrak{h}^{*} \rightarrow a d_{L} \mathfrak{h}^{*}: U \rightarrow a d_{L} U$ is injective since $L$ is semi-simple; thus if $\mathfrak{h}^{*}$ is semi-simple the trace form $\operatorname{tr} a d_{L} U a d_{L} V=K(U, V)$ is non-degenerate. Next if $\mathfrak{h}^{*}=R J \oplus \mathfrak{h}^{* \prime}$ it suffices to show $\operatorname{tr}\left(a d_{L} J\right)^{2} \neq 0$. But a straight forward computation shows $\left(a d_{L} J\right)^{2}$ has minimum polynomial $x^{2}+4 x \in R[x]$ and therefore has non-zero trace. Thus in both cases $\mathfrak{h}^{*} \cap \mathfrak{h}^{* \perp}=0$ so we can decompose $L=M+\mathfrak{h}^{*}$ with $M=\mathfrak{h}^{* \perp}$. Also $K \mid M \times M$ is a nondegenerate invariant form relative to the multiplication $P \cdot Q=[P, Q]_{M}$ in $M$.

If $M \cdot M \neq 0$ and $M$ is not simple, then $M$ has a proper minimal adh*invariant ideal $N$. Since $K$ is a nondegenerate invariant form on $M$ we 
have that $N^{\perp} \subset M$ is an ad $\mathfrak{h}^{*}$-invariant ideal. Thus $N \cap N^{\perp}$ is an ad $\mathfrak{h}^{*}$ invariant ideal in $M$ so that $N \cap N^{\perp}=N$ or $N \cap N^{\perp}=0$. In case $M \cdot M=0$ use a proper minimal ad $\mathfrak{h}^{*}$-invariant subspace $N \subset M$.

Case $N \cap N^{\perp}=N$ is analogous to the same situation in [13, section 3]. Thus since $a d \mathfrak{h}^{*}$ is completely reducible on $M$ we can find an ad $\mathfrak{h}^{*}$ invariant complement $N^{\prime}$ and write $M=N+N^{\prime}$. Since $K(N, N)=0$ it suffices to show $K\left(N, N^{\prime}\right)=0$ to show $N=0$.

To use the methods in $[11 ; 13]$ it suffices by $[13$, section 4] to show $\operatorname{tr} a d_{\mathfrak{h}^{*}} \mathfrak{h}^{*}=0$, which is clear, and $\operatorname{tr} a d_{N} \mathfrak{h}^{*}=0$ for any ad $\mathfrak{h}^{*}$-invariant subspace $N$. This is also clear from what have proved: if $\mathfrak{h}^{*}=R J \oplus \mathfrak{h}^{* \prime}$ then $a d_{L} J$ minimum polynomial $x^{3}+4 x$ on $N$ so that $\operatorname{tr}\left(a d_{N} J\right)=\operatorname{tr}_{N}\left(a d_{L} J\right)=0$; also since $\mathfrak{h}^{* \prime}$ is semi-simple and $\mathfrak{h}^{* \prime} \rightarrow a d_{N} \mathfrak{h}^{* \prime}$ is a representation, $\operatorname{tr}\left(a d_{N} \mathfrak{h}^{* \prime}\right)=0$. Thus as in [13], we obtain for $P \in N, Q \in N^{\prime}$ that

$$
K(P, Q)=2 \operatorname{tr}_{N} \sigma(P, Q)
$$

where for $R \in M, \sigma(P, Q) R=[h(R, Q), P]$ with $h(R, Q)=[R, Q]_{\mathfrak{h}^{*}} \in \mathfrak{h}^{*}$. But computations show $\operatorname{tr}_{N} \sigma(P, Q)=\operatorname{tr} a d_{N} h(P, Q)=0$ so that $K\left(N, N^{\prime}\right)=0$. In case $M \cdot M=0$, then just choose $N$ to be a minimal $\mathfrak{h}^{*}$-invariant subspace of $M$ and the formulas in [13] simplify because the mappings $L(P): M$ $\rightarrow M: Q \rightarrow P \cdot Q$ are zero. Thus any minimal $\mathfrak{h}^{*}$-invariant subspace of $M$ is non-isotropic which is just the other case.

Case $N \cap N^{\perp}=0$ where $N$ is any minimal ad $\mathfrak{h}^{*}$-invariant ideal of $M$ (if $M \cdot M \neq 0$ ). Using Killing form arguments as in Lemma 3 of [13] we have $\left[N, N^{\perp}\right]=0$ and computing as in Lemma 2 of [13] we see that $L_{1}=N$ $\dot{+} h(N, N)$ is an ideal in $L$ where $h(N, N)=\mathfrak{h}_{1}^{*}$ is the subalgebra of $\mathfrak{h}^{*}$ generated by all $h(R, S)$ for $R, S \in N$. Next note that $K \mid L_{1} \times L_{1}$ is nondegenerate so we can decompose $L=L_{1} \oplus L_{1}{ }^{\perp}$. Now if $L_{1}{ }^{\perp} \subset \mathfrak{h}^{*}$, then $M=N$. If $M$ is not a zero algebra, then it is a simple algebra relative to the multiplication $P \cdot Q=[P, Q]_{M}$; otherwise if $M$ has a proper ideal it has a proper ad $\mathfrak{h}^{*}$-invariant ideal, contrary to the choice of $N$. Now if $L_{1}^{\perp} \not \subset \mathfrak{h}^{*}$, then $L_{1} \perp$ satisfies the same conditions as $L$ and we continue by induction to obtain part (b). If $M \cdot M=0$, then replace the "minimal ideal $N$ " by a "minimal ad $\mathfrak{h}^{*}$-invariant subspace $N$ of $M$ ". In both cases the $\mathfrak{h}_{i}^{*}$ is reductive in $\mathfrak{h}^{*}$ since it is an ideal and therefore radical $\mathfrak{h}_{i}^{*}=\mathfrak{h}_{i}^{*} \cap$ radical $\mathfrak{h}^{*}$ which is at worst $R J$. The example in [13, section 4] shows the case $L_{i}$ semi-simple cannot be reduced to the case $L_{i}$ simple. 


\section{BIBLIOGRAPHY}

[1] S. Helgason: Differential Geometry and Symmetric Spaces, Academic Press, 1962.

[2] N. Jacobson: Lie Algebras, John Wiley, 1962.

[3] N. Jacobson: Structure of Rings, American Mathematical Society, 1956.

[4] N. Jacobson: Completely reducible Lie algebras of linear transformations, Proc. Amer. Math. Soc., Vol. 2 (1951), 105-113.

[ 5 ] B. Kostant: On holonomy and homogeneous spaces, Nagoya Math. Jol., Vol. 12 (1957), $31-54$.

[6] O. Loos: Thesis, University of Munich, 1966.

[ 7 ] K. Nomizu: Recent development in the theory of connections and holonomy groups, Advances in Mathematics, Vol. 1, Academic Press, 1961.

[ 8 ] K. Nomizu: Invariant affine connections on homogeneous spaces, Amer. Math. J., Vol. 76 (1954), 33-65.

[9] K. Nomizu: Studies on Riemannian homogeneous spaces, Nagoya Math. J., Vol. 9 (1955), 43-56.

[10] A. Sagle: On anti-commutative algebras and homogeneous spaces, to appear J. of Math. and Mech., 1967.

[11] A. Sagle: A note on simple anti-commutative algebras obtained from reductive homogeneous spaces, to appear in Nagoya Math. J.

[12] A. Sagle: A note on triple systems and totally geodesic submanifolds in a homogeneous space, to appear in Nagoya Math. J.

[13] A. Sagle and D.J. Winter: On homogeneous spaces and reductive subalgebras of simple Lie algebras, to appear in Trans. Amer. Math. Soc.

[14] R.D. Schafer: Inner derivations of nonassociative algebras, Bull. Amer. Math. Soc., Vol. 55 (1949), 769-776.

[15] J. Wolf: Complex homogeneous contact manifolds and quaternionic symmetric spaces, J. of Math. and Mech., Vol. 14 (1965), 1033-1042.

[16] J. Wolf: The geometry and structure of isotropy-irreducible homogeneous spaces, to appear.

[17] K. Yamaguti: Note on Malcev algebras, Kumamoto J. Sci., Vol. 5 (1962), 171-184.

[18] J. Hano and Y. Matsushima: Some studies on Kaehlerian homogeneous spaces, Nagoya Math. J., Vol. 11 (1957), 77-92.

\section{University of Minnesota}

